\title{
A theoretical study of the liquid structure of nitromethane with RISM method
}

\author{
$\operatorname{AUTHOR}(\mathrm{S})$ :
}

Hayaki, Seigo; Sato, Hirofumi; Sakaki, Shigeyoshi

\section{CITATION:}

Hayaki, Seigo ...[et al]. A theoretical study of the liquid structure of nitromethane with RISM method. Journal of Molecular Liquids 2009, 147(1-2): 9-12

\section{ISSUE DATE:}

2009-07-20

URL:

http://hdl.handle.net/2433/123549

\section{RIGHT:}

Copyright (c) 2008 Elsevier B.V.; This is not the published version. Please cite only the published version.; この論文は出版社版でありません。引 用の際には出版社版をご確認ご利用ください。 


\title{
A Theoretical Study of the Liquid Structure of Nitromethane with RISM Method
}

\author{
Seigo Hayaki, Hirofumi Sato*, Shigeyoshi Sakaki \\ Department of Melecular Engineering, Kyoto University, Kyoto Daigaku Katsura, \\ Kyoto 615-8510, Japan
}

\begin{abstract}
An integral equation theory for molecular liquids, reference interaction site model (RISM), was applied to understand the liquid structure of nitromethane, which is extensively used as a solvent. In the theory, liquid structure is described by a set of pair correlation functions. With the aid of ab initio molecular orbital calculations, representative peaks were able to be related to specific configurations of molecules.
\end{abstract}

Key words: reference interaction site model, nitromethane, liquid structure, radial distribution function

\section{Introduction}

Nitromethane is one of the simplest nitro-compounds and is liquid at ordinary condition. The substance is extremely versatile: Not only a typical solvent used for cleaning process and reaction media but also an intermediate in organic

* Corresponding author. FAX: +81-75-383-2799

Email address: hirofumi@moleng.kyoto-u.ac.jp (Hirofumi Sato). 
synthesis of various chemicals. The liquid has been extensively used for many industrial applications deeply related to our lives. Furthermore, nitromethane is known as its explosive properties: it burns easily and used as fuel. Due to its high-performance and reduced-toxicity, it has been known as a storable monopropellant having a prospects of use for space thrusters [1]. Because of these factors, the cellular structure of detonations in liquid nitromethane [2], and burning behaviour have been reported by means of computational modelling. But the molecular level information of the liquid has not been provided from these studies.

Concerning physicochemical character of this molecule, it is highly polar solvent ( $\varepsilon=37.5)$, which is slightly smaller than dimethyl sulfoxide (47.2), glycerol (46.5) and comparable to acetonitrile (36.6). A high $\varepsilon$ may attribute to higher dipole moment of the molecule $(\mu=3.46 \mathrm{D})$. It is conceivable that the nitro group draws electron and this intramolecular charge-deviation is responsible for the intermolecular interaction. It has been argued that the existence of the hydrogen boding between $\mathrm{C}-\mathrm{H} \cdots \mathrm{O}-\mathrm{N}$ in liquid nitromethane. At the same time, it is likely that the dispersion interaction between nitro groups might also contribute to the characterization of the liquid structure. Very recently, a direct observation of the liquid structure was achieved by x-ray and neutron diffraction, together with Car-Parrinello simulation [3]. There are also a few molecular simulation studies [4-6], but the detailed characterization of the liquid structure is still unclear.

Reference interaction site model (RISM) is an integral equation theory for molecular liquids $[7,8]$. Unlike molecular simulation methods such as molecular dynamics and Monte Carlo, the integration over the configurational space of liquid in RISM theory is performed in an analytic way and the computa- 
tional cost is dramatically reduced compared to the simulations. It is more important that integral equation theory can offer constructional and systematic understanding of solvation phenomena. RISM has been also combined with $a b$ initio molecular orbital (MO) theory (RISM-SCF) [9-11]. It determines the electronic structure of a solute and the solvent distribution around it in a self-consistent manner, successfully applied to various chemical processes in solution phase such as organometallic reaction in solution phase [12].

In the present study, the liquid structure of nitromethane is discussed by means of RISM theory. Results from ab initio MO computations are also incorporated to analyze the liquid structure. Note that RISM and MO computations were separately employed in this study.

\section{Method}

In this work, two set of parameters ( "Set A" and "Set B") were examined for the intermolecular interaction. One of them is the same as that in our previous study [12], where nitromethane was used as solvent. The standard Lennard-Jones 12-6 potential plus Coulombic interaction was used and the parameters were determined from the OPLS parameter set [13] and from the set reported by Alper et al. [5].

$$
E=\frac{q q^{\prime}}{r}+4 \epsilon\left[\left(\frac{\sigma}{r}\right)^{12}-\left(\frac{\sigma}{r}\right)^{6}\right]
$$

The venerable Lorentz-Berthelot mixing rule $\left(\sigma_{A B}=\left(\sigma_{A}+\sigma_{B}\right) / 2\right.$ and $\epsilon_{A B}=$

$\left.\sqrt{\epsilon_{A} \epsilon_{B}}\right)$ was employed to construct the potential as collected in Table 1 . The main difference between the two is the charge assigned on carbon atom. 
The number density $(\rho)$ was assumed to be 0.0112464 molecules $/ \AA^{3}$, equal to the experimental value $\left(1.14 \mathrm{~g} / \mathrm{cm}^{3}\right)$, and temperature was set to 298.15 K. RISM calculations were carried out to obtain the structure of liquid nitromethane on a grid of 4096 points using the hyper netted chain (HNC) closure approximation. In the RISM calculation, the rotation of methyl group around $\mathrm{C}-\mathrm{N}$ bond can not be treated and the dihedral angles of $\mathrm{H}-\mathrm{C}-\mathrm{N}-\mathrm{O}$ are fixed at $0^{\circ}\left(\mathrm{H}_{\mathrm{A}}\right)$ and $\pm 120^{\circ}\left(\mathrm{H}_{\mathrm{B}}\right)$. As shown below, however, the rigid-molecule approximation seems to have no affect on the discussion. All the RISM computations were performed by our own developing program package.

On the analysis of liquid structure obtained from RISM computations, standard ab initio MO computations were performed by using the Gaussian 03 suite of programs [14]. Several geometrical structures of nitromethane dimer were optimized at MP2 level using $6-31++\mathrm{G}^{* *}$ basis set.

\section{Results and Discussion}

\subsection{Optimized Structure of Dimer}

To understand the mutual orientation of nitromethane molecules, standard $a b$ initio MO calculations of the dimer system were performed. Two optimized structure (I and II) were obtained in gas phase as shown in Fig. 1. Structure I is antiparallel arrangement of the dimer, while in structure II nitromethanes arrange in head-to-tail conformation. The potential energy difference between them is not so large and the structure I is more stable than II by $3.88 \mathrm{kcal} / \mathrm{mol}$. The free energy difference between them is further small, no more than 0.52 $\mathrm{kcal} / \mathrm{mol}$. The reduction of the free energy difference arises from entropic con- 
tribution, especially from vibrational correction term. Li and co-workers have been reported a DFT (B3LYP) study for the same dimer [15], in which the four different structures were shown as possible stable geometries. In our case, optimization by MP2 method eventually arrive at the aforementioned one of two structures even when starting from their reported structures. Megyes et al. also showed optimization result [3], but only the structure I was reported.

\subsection{Structure of Liquid Nitromethane}

In the framework of RISM theory, solvation structure is described in terms of radial distribution functions (RDFs). Note that the same function is also called as pair correlation function $(\mathrm{PCF})$ because site-site pair is treated in RISM theory. The PCF represents the probability to find two atoms at a distance $r$ and provides insight into the specific interactions in liquids. The PCF computed with Set A is shown in Fig. 2. The profiles of the function resemble the results of previous simulation studies [3-6]. The interatomic distances in these optimized structures are indicated in the figure with the white arrows (I) and black arrows (II). Almost all peak positions in PCFs coincide with the interatomic distances either one or two optimized structures mentioned above.

\subsubsection{The $C-C P C F$}

The $\mathrm{C}-\mathrm{C}$ PCF rises around $3.0 \AA$ and gradually reaches the bleary peak (5.2 $\AA$ ). A shoulder is also seen around $4.0 \AA$. In the optimized structures, distances between two carbon atoms are respectively 4.6 and $5.1 \AA$, being slightly deviated from the PCF peak. 
Compared to the previous simulations, the height of the shoulder look slightly lower. In the present work, there are two sources that makes the PCFs different from the previous ones. One is to perform RISM, in which different concept and procedure are used from standard molecular simulations. The statistical ensemble in RISM is appropriate but the theory is, at the same time, an approximation because the HNC is regarded as a truncated form of the $\log \left[\rho^{(1)}(1 \mid \phi) / z^{*}(1)\right]$ expansion [16]. The other is a difference in the parameter set describing the solvent. In Figure 3, the same PCFs by using Set B are plotted [5]. The positions of peaks are not changed and liquid structures look very similar. However, the peak heights are slightly elevated at $\mathrm{C}-\mathrm{O}$ and C-N pairs compared to Set A. The shoulder of C-C pair at $4.0 \AA$ is obviously enhanced.

By integrating the $\mathrm{C}-\mathrm{C}$ PCF up to the first minimum, the coordination number is computed as 14.1 both from Set A and B, which shows reasonable agreement with the previous report by Sorescu and co-workers; 12.8 [6]. Note that their parameters were originally prepared for crystalline nitromethane.

\subsubsection{The $\mathrm{O}-\mathrm{H} P C F$}

O-H PCF shows a peak around $2.5 \AA$ (Fig. 2), which is close to the sum of Lennard-Jones parameters, $\left(\sigma_{\mathrm{O}}+\sigma_{\mathrm{H}}\right) / 2=2.73 \AA$. This indicates that oxygen and hydrogen atoms are directly contacted, but the peak height is lower than that of typical hydrogen bonding found in protic solvents. The same interaction in Set B (Fig. 3) is more drastic and the peak height is less than 1.0. One might consider that the weak interaction between oxygen and hydrogen in Set A mainly attributes to the small charge assigned on hydrogen atom 
$(+0.06|e|$, see Table 1$)$. However, the charge on hydrogen is much greater in Set $\mathrm{B}(+0.142|e|)$ and a repulsive interaction between oxygen atom $(-0.406|e|)$ and negatively charged carbon $(-0.331|e|)$ should be the reason to weaken the $\mathrm{O}-\mathrm{H}$ interaction. The second peak of $\mathrm{O}-\mathrm{H}$ PCF $(4.2 \AA)$ is assinged to the contribution from the other oxygen atom in nitro group $(4.1 \AA$ in I). The $\mathrm{O}-\mathrm{H}$ pair distance is longer than a typical hydrogen-bonding even for the first peak, consequently indicating that hydrogen bonding is very weak nor does not exist in this system, independently of the parameters. In the present study, the rotation around $\mathrm{C}-\mathrm{N}$ bond was not treated. From the PCFs, the two sets of hydrogen atoms $\left(\mathrm{H}_{\mathrm{A}}\right.$ and $\left.\mathrm{H}_{\mathrm{B}}\right)$ are virtually equivalent and effect from the rotation is not significant.

Then, is the electrostatic interaction not important? Figure 4 compares the normal PCFs and those computed by removing all the charges in Set A. Note that since the two different $\mathrm{O}-\mathrm{H}$ PCFs are very similar, only $\mathrm{H}_{\mathrm{B}}$ PCFs are shown for brevity. While $\mathrm{C}-\mathrm{O}$ and $\mathrm{O}-\mathrm{H}$ PCFs are not changed by turning off the electrostatic interaction, the peak positions are drastically changed in $\mathrm{C}-\mathrm{N}$ and $\mathrm{C}-\mathrm{C}$ PCFs. This result suggests that the electrostatic interaction between heavy atoms is primarily important to determine the liquid structure. Remember that the DFT computes several bound structures but some of them disappear in MP2 computations, which can treat dispersion interaction. This paradoxical result might suggest the importance of electrostatic interaction in the present nitromethane system.

By the recently performed x-ray diffraction study by Megyes et al., the experimental RDF is available [3]. Fig. 5 shows the comparison between the RDF and the present RISM calculation. Using the same scattering weighting factors (C-C, 0.04; C-N, 0.08; C-O, 0.18; N-O, 0.21; N-N, 0.05; and O-O, 0.25), 
total RDF $(g(r))$ was computed by averaging over PCFs obtained from RISM theory. The contributions from hydrogen atoms were omitted. Both of RDFs show a good agreement though a very slight difference is seen in the shoulder around $2.5 \AA$.

Before closing the section, we would like to call attention to the readers that the analysis based on dimer cluster is just an expedient: we never support the existence of the association pair with certain life time. In reality, all the molecules move around and the "clusters" - regardless of whether it can be meaningfully defined - shown in the figures would disappear immediately. Actually, the atomic distances are not perfectly fit to the peak positions due to the contribution from molecular fluctuation. But the analysis through the optimized structure could offer pictorial understanding of liquid structure.

\section{Concluding Remarks}

The liquid structure of nitromethane was studied by means of RISM theory. The obtained solvation structures, namely RDFs, are consistent with the previous simulation studies. The reliability of the present computation can be also confirmed by the agreement with the experimentally obtained RDF. We have examined two sets of intermolecular parameters. Although some differences were seen in PCFs, the coordination number and total RDF were almost identical in both of the parameter sets.

With the aid of the optimized structures computed by ab initio MO method (MP2), the peaks of RDFs can be clearly assigned. We found that antiparallel arrangemant and head-to-tail forms can be regarded as representative con- 
formation. As previously pointed out, hydrogen bonding is weak or does not exist in liquid nitromethane.

\section{Acknowledgements}

This work was financially supported by Grant-in-Aids on basic research (No. 15350012), Priority Areas for "Molecular Theory for Real Systems" (No.461), Creative Scientific Research, NAREGI project from the Ministry of Education, Science, Sports, and Culture. 
Table 1

Lennard-Jones parameters for nitromethane solvent.

\begin{tabular}{c|ccc|ccc}
\hline \hline & \multicolumn{3}{|c|}{ Set A } & \multicolumn{3}{c}{ Set B } \\
\hline Atom & $\sigma / \AA$ & $\epsilon / \mathrm{kcal} \mathrm{mol}^{-1}$ & $q /|e|$ & $\sigma / \AA$ & $\epsilon / \mathrm{kcal} \mathrm{mol}^{-1}$ & $q /|e|$ \\
\hline $\mathrm{N}$ & 3.250 & 0.120 & 0.540 & 3.562 & 0.200 & 0.717 \\
$\mathrm{O}$ & 2.960 & 0.170 & -0.370 & 2.999 & 0.120 & -0.406 \\
$\mathrm{C}$ & 3.500 & 0.066 & 0.020 & 3.809 & 0.080 & -0.331 \\
$\mathrm{H}$ & 2.500 & 0.015 & 0.060 & 2.327 & 0.022 & 0.142 \\
\hline \hline
\end{tabular}



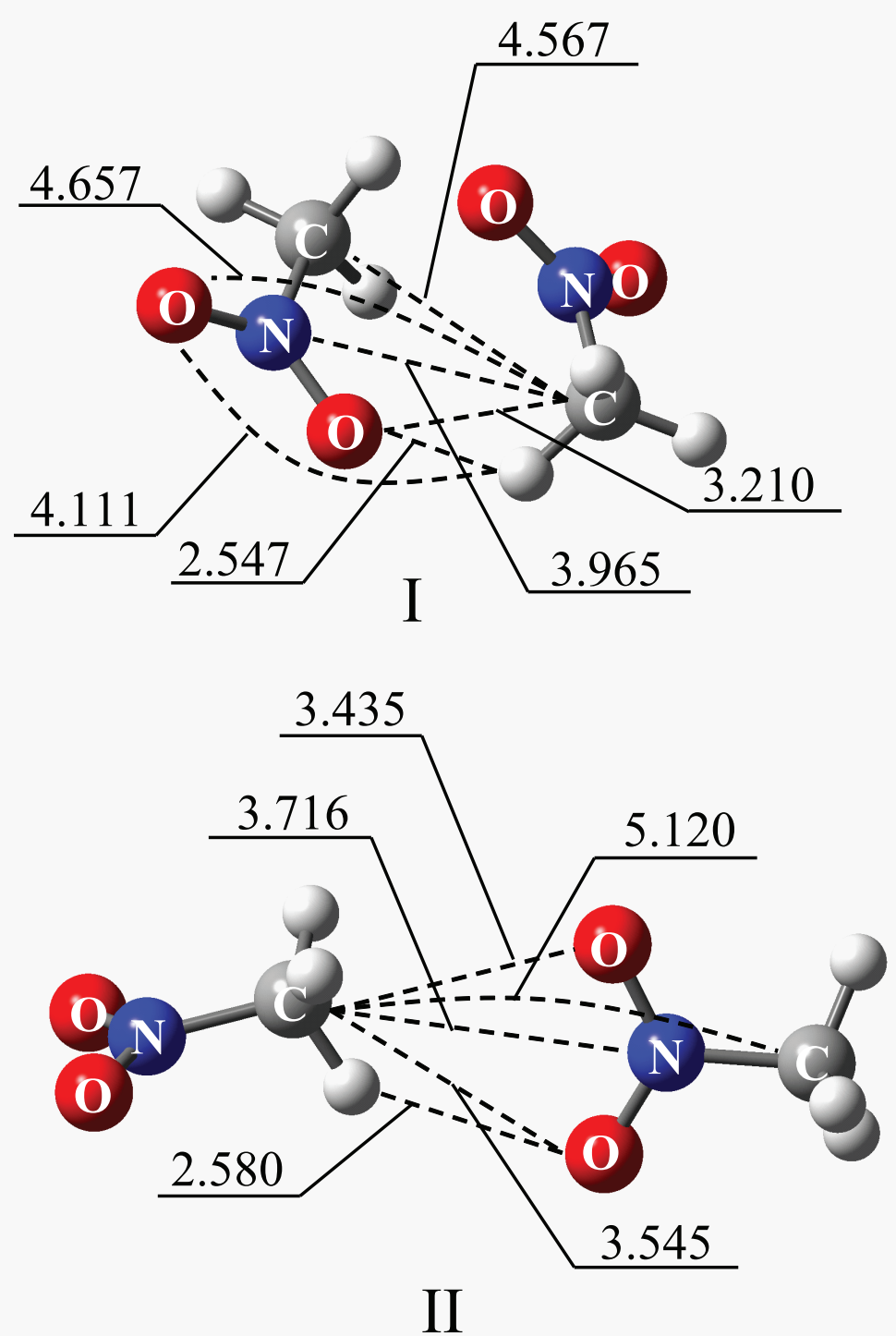

Fig. 1. Optimized structures of nitromethane dimers. I and II are the antiparallel arrangement and head-to-tail conformation, respectively. Important interatomic distances are given in $\AA$. 


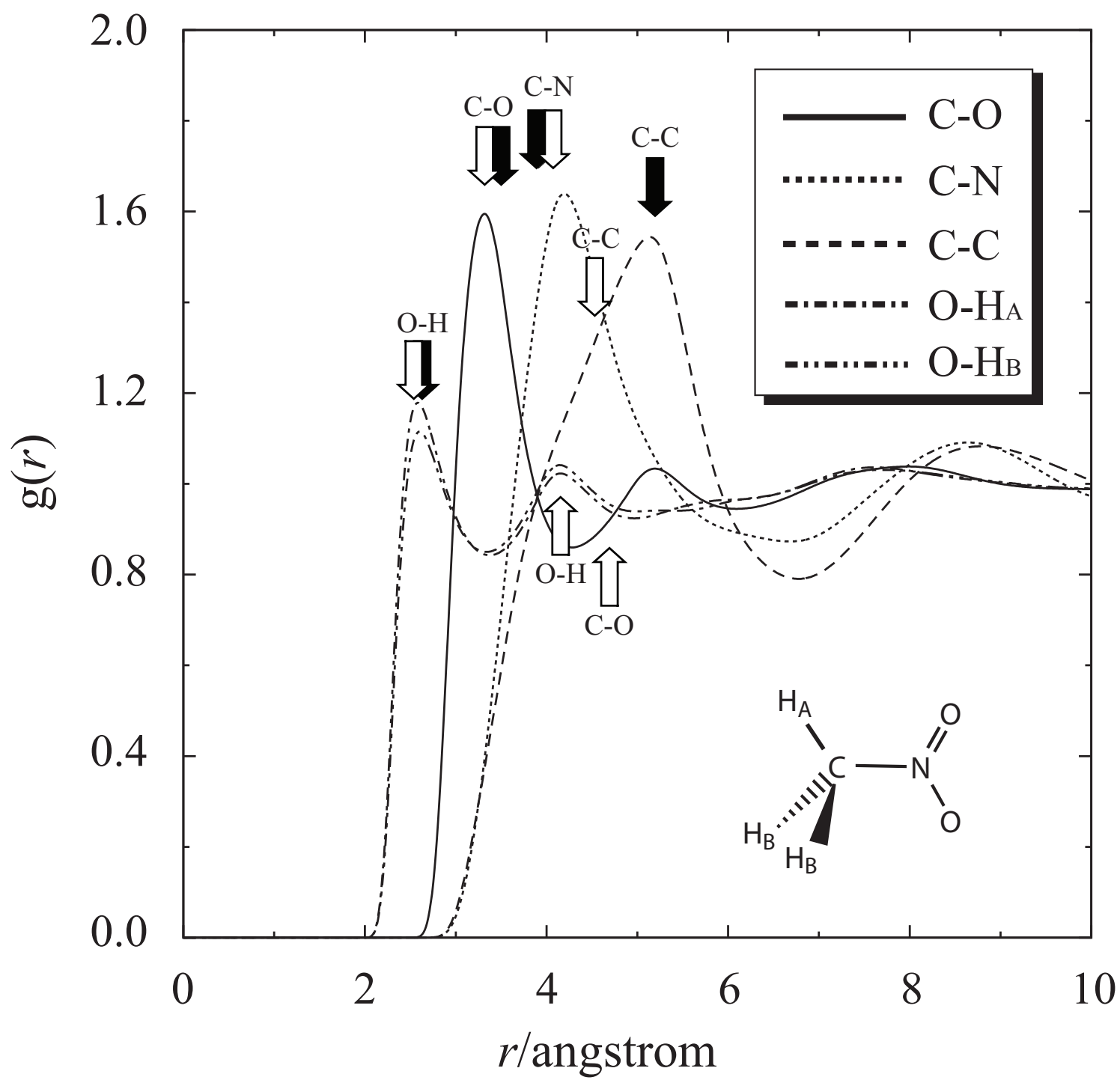

Fig. 2. PCFs for the $\mathrm{C}-\mathrm{O}, \mathrm{C}-\mathrm{N}, \mathrm{C}-\mathrm{C}$, and $\mathrm{O}-\mathrm{H}$. White arrows and black arrows indicate the interatomic distances in structure I and structure II, respectively. See text for details. 


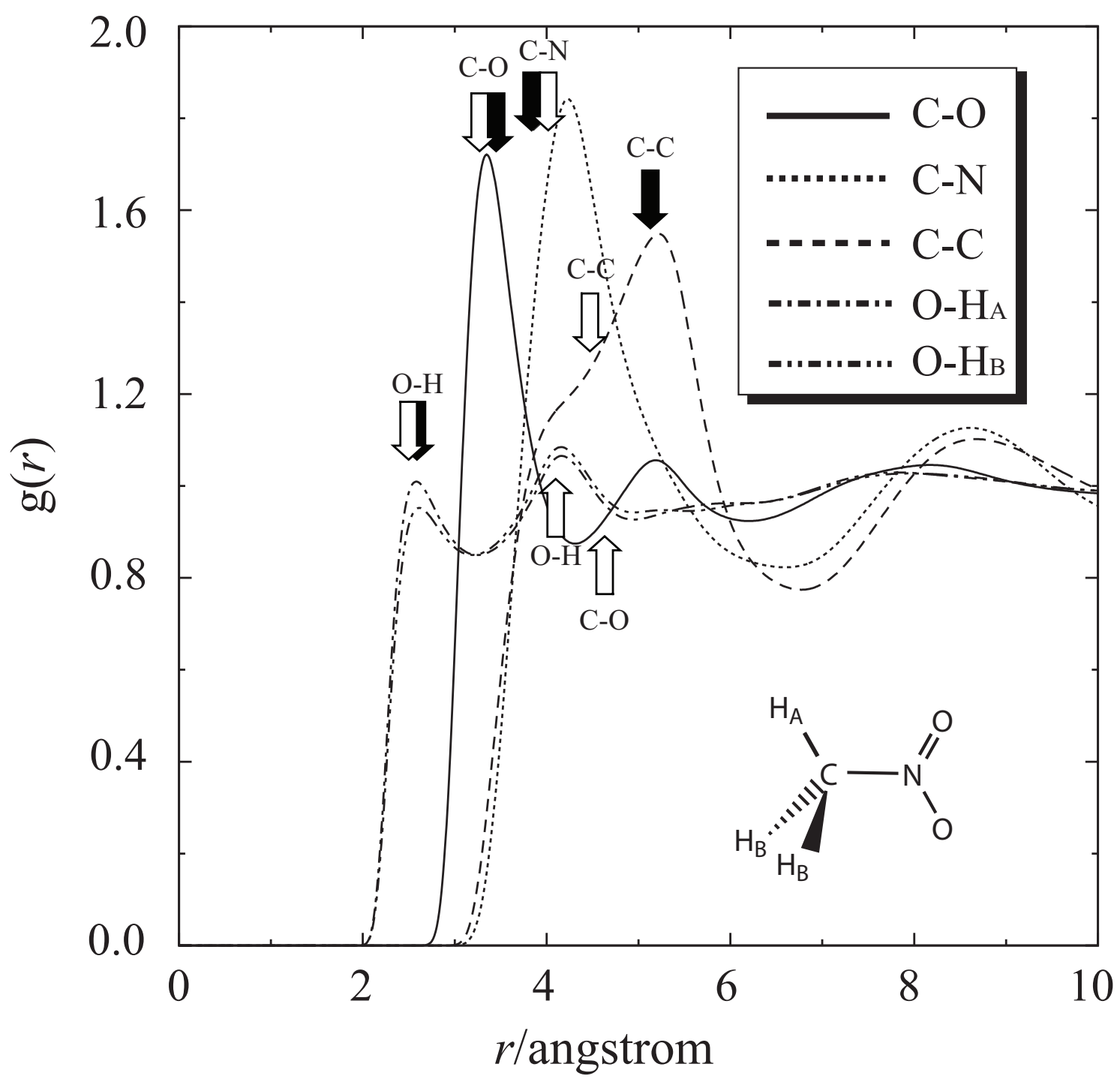

Fig. 3. PCFs for the $\mathrm{C}-\mathrm{O}, \mathrm{C}-\mathrm{N}, \mathrm{C}-\mathrm{C}$, and $\mathrm{O}-\mathrm{H}$ computed with the parameter set by Alper et al. [5] (Set B). See the caption in Fig. 2 


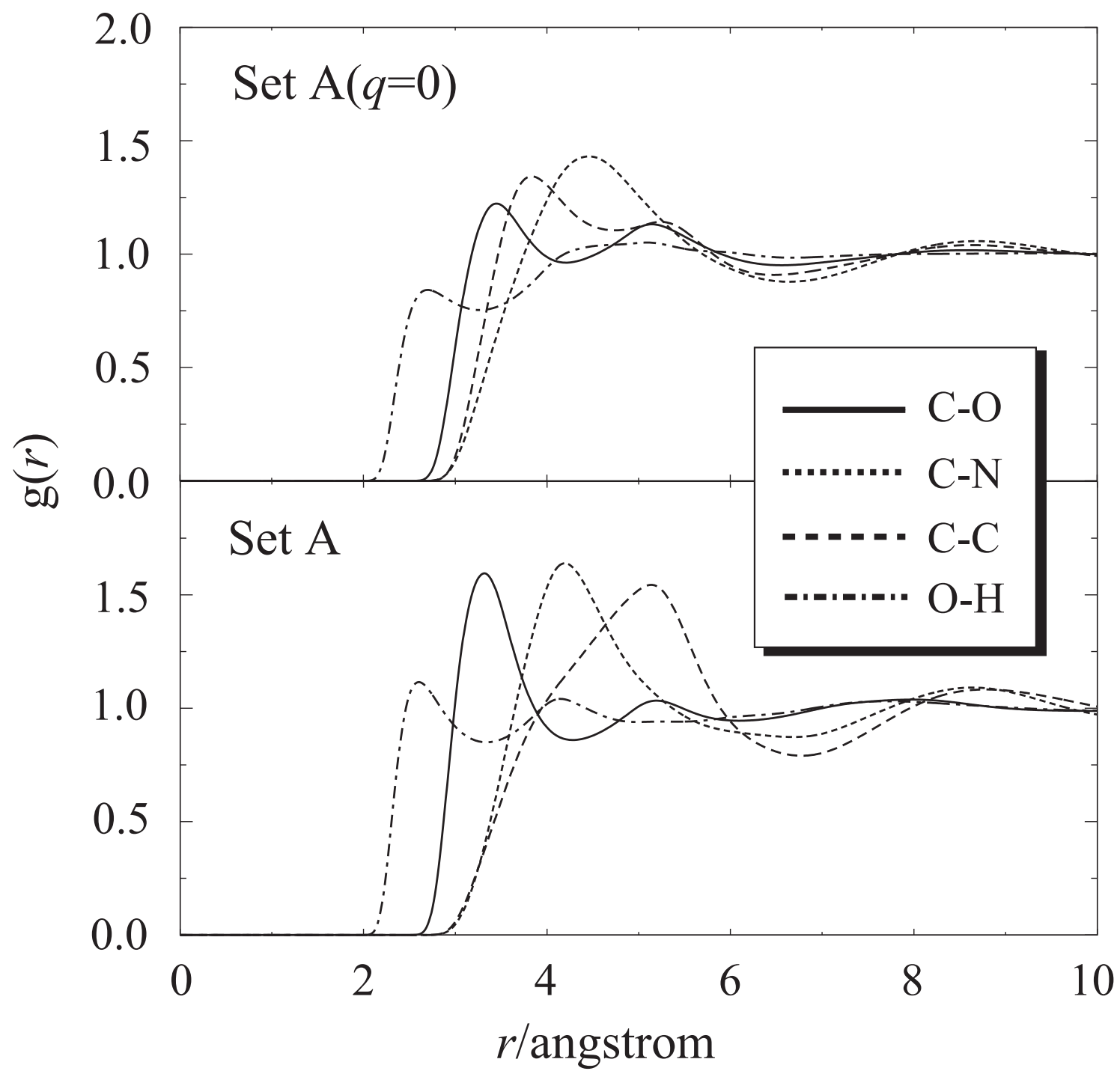

Fig. 4. PCFs for the $\mathrm{C}-\mathrm{O}, \mathrm{C}-\mathrm{N}, \mathrm{C}-\mathrm{C}$, and $\mathrm{O}-\mathrm{H}$ computed with (lower panel) Set A and (upper panel) all the charges were removed. 


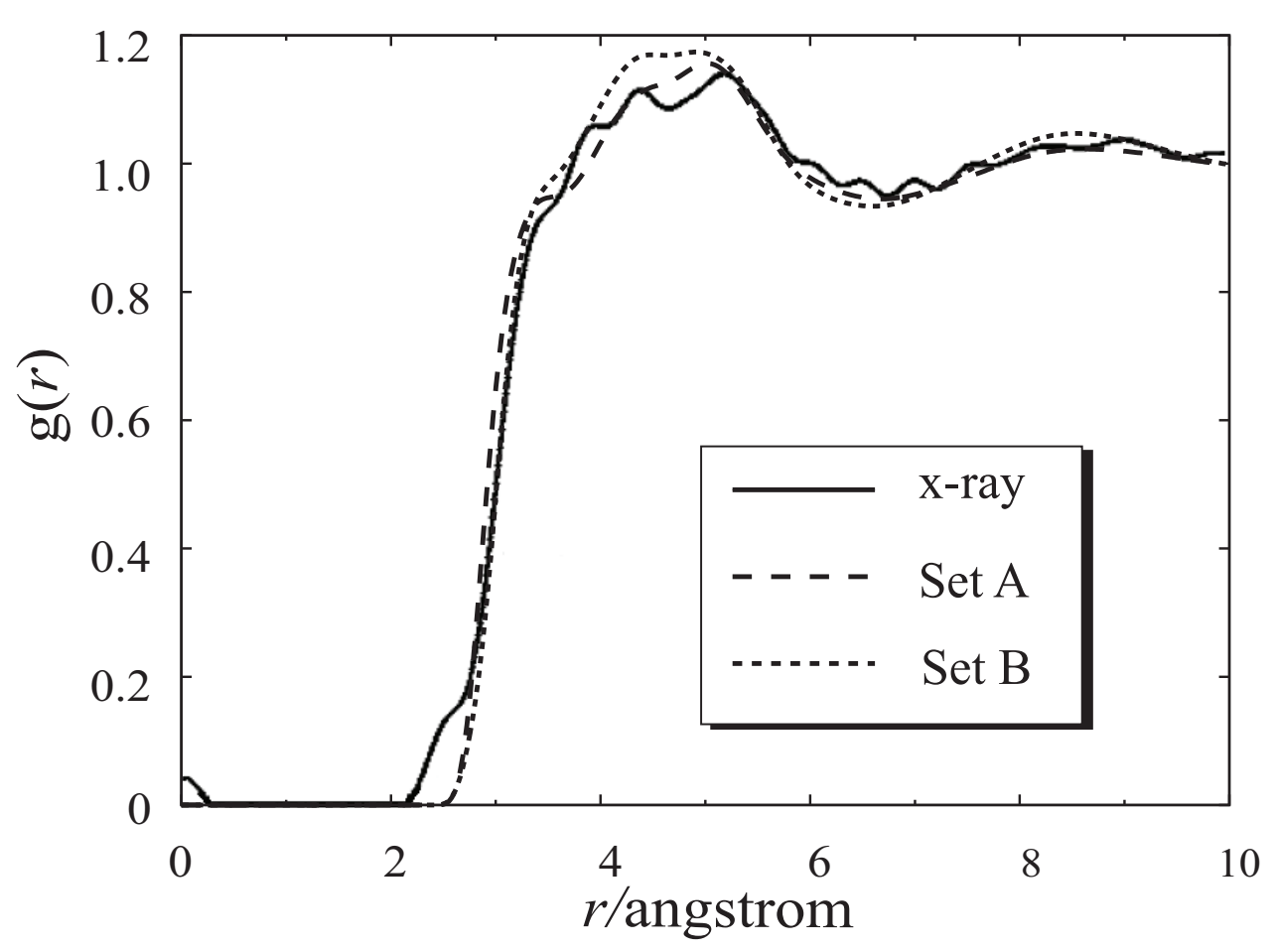

Fig. 5. Intermolecular total radial distribution functions obtained by x-ray diffraction (solid line) and RISM calculations with set A (dashed line) and set B (dotted line). 


\section{References}

[1] E. Boyer, K. K. Kuo, Proc. Combust. Inst. 31 (2007) 2045.

[2] E. S. Oran, K. Kailasanath, Combust. Flame 65 (1986) 339.

[3] T. Megyes, S. Bálint, T. Grósz, T. Radnai, I. Bakó, J. Chem. Phys. 126 (2007) 164507.

[4] J. M. Seminario, M. C. Concha, P. Politzer, J. Chem. Phys. 102 (1995) 8281.

[5] H. E. Alper, F. Abu-Awwad, P. Politzer, J. Phys. Chem. B 103 (1999) 9738.

[6] D. C. Sorescu, B. M. Rice, D. L. Thompson, J. Phys. Chem. A 105 (2001) 9336.

[7] D. Chandler and H. C. Andersen, J. Chem. Phys. 57 (1972) 1930.

[8] F. Hirata, P. J. Rossky, Chem. Phys. Lett. 83 (1981) 329.

[9] S. Ten-no, F. Hirata, S. Kato, J. Chem. Phys. 100 (1994) 7443.

[10] H. Sato, F. Hirata, S. Kato, J. Chem. Phys. 105 (1996) 1546.

[11] D. Yokogawa, H. Sato, S. Sakaki, J. Chem. Phys. 126 (2007) 244054.

[12] S. Hayaki, D. Yokogawa, H. Sato, S. Sakaki, Chem. Phys. Lett. 458 (2008) 329 .

[13] M. L. P. Price, D. Ostrovsky, W. L. Jorgensen, J. Comput. Chem. 22 (2001) 1340.

[14] Gaussian 03, Revision D.02; J. A. Pople et al., Gaussian, Inc., Wallingford CT. 
[15] J. Li, F. Zhao, F. Jing, J. Comput. Chem. 24 (2003) 345.

[16] For example; J.-P. Hansen, I. R. McDonald, Theory of Simple Liquids, Elsevier Academic Press 1986. 